\title{
Inflammatory myofibroblastic tumor of the mediastinum presenting as superior vena cava syndrome
}

\author{
Masafumi Yamaguchi, MD, PhD, ${ }^{a}$ Ichiro Yoshino, MD, PhD, atsushi Osoegawa, MD, ${ }^{\text {a }}$ Toshifumi Kameyama, $\mathrm{MD}^{\text {a }}$ \\ Tetsuzo Tagawa, MD, ${ }^{\text {a }}$ Seiichi Fukuyama, MD, PhD, ${ }^{\text {a }}$ Yoshinao Oda, MD, PhD, ${ }^{\text {b }}$ Masazumi Tsuneyoshi, MD, PhD, ${ }^{\mathrm{b}}$ and \\ Yoshihiko Maehara, MD, PhD ${ }^{\text {a }}$ Fukuoka, Japan
}

I nflammatory myofibroblastic tumor (IMT) is a rare neoplasm that originates in the soft tissues and is composed of distinctive pseudosarcomatous inflammatory lesion with spindle cell proliferation. ${ }^{1}$ The age of the patients varied; however, many of them are children or young adults with an average age of 9.7 years. ${ }^{1}$ The clinical symptoms depend on the location of the tumor. ${ }^{2-6}$ Although IMTs have been reported in the mediastinum, heart, ${ }^{1}$ lung, ${ }^{7}$ and trachea ${ }^{8}$ as an intrathoracic tumor, cases of superior vena cava (SVC) syndrome have been extremely uncommon. We herein report a case of IMT in the mediastinum presenting as SVC syndrome.

\section{Clinical Summary}

A 31-year-old woman with no subjective symptoms was found to have a mediastinal mass by annual routine chest radiograph on June 25,2002 . She was referred to our department on July 19 , 2002, for further surgical treatment. At the first presentation, dilatation of the right external jugular vein was remarkable, but the patient's upper extremities were not swollen. A chest computed tomogram (CT) revealed a huge anterior mediastinal mass, measuring $13.5 \times 10.5 \times 6.0 \mathrm{~cm}$, with heterogeneous and minor calcification inside. The bilateral brachiocephalic veins (BCVs), SVC, and the trachea were compressed by the tumor. Although the tumor was located near the proximal site of the brachiocephalic artery (BCA), aortic arch, left common carotid artery (CCA), and left subclavian artery (SA), no obvious invasion to the arterial walls was identified (Figure 1,A). Chest magnetic resonance imaging also showed that the BCA was surrounded by the tumor, and the aortic arch, the left CCA, and the left SA were closely adjacent to the tumor. Venography via the subcutaneous veins of the bilateral upper extremities showed severe narrowing of the right $\mathrm{BCV}$ and occlusion from the left $\mathrm{BCV}$ to the SVC with collateral venous drainage via the inferior thymic vein and the azygos vein (Figure 1, $B$ ). The patient's upper extremities became

From the Departments of Surgery and Science ${ }^{\mathrm{a}}$ and Anatomic Pathology, Graduate School of Medical Sciences, Kyushu University, Fukuoka, Japan.

Received for publication March 11, 2003; accepted for publication April 2, 2003.

Address for reprints: Masafumi Yamaguchi, MD, PhD, Department of Surgery and Science, Graduate School of Medical Sciences, Kyushu University, 3-1-1 Maidashi, Higashi-ku, Fukuoka 812-8582, Japan (E-mail: masafumi@surg2.med.kyushu-u.ac.jp).

J Thorac Cardiovasc Surg 2003;126:870-2

Copyright $\odot 2003$ by The American Association for Thoracic Surgery $0022-5223 / 2003 \$ 30.00+0$

doi:10.1016/S0022-5223(03)00611-1

swollen and her bilateral jugular vein dilated further during the 2-week observation period before the operation.

Surgery consisting of extirpation of the tumor with a combined resection of the SVC and a total aortic arch replacement under cardiopulmonary bypass (CPB) with selective normograde cerebral perfusion was planned on August 12, 2002. A median sternotomy was performed. The tumor was not invasive to the arterial walls, and without intervention, it soon would have dissected from the adventitia of the aorta and BCA. The pericardium was opened and the apex of the auricle of the right atrium and left BCV was bypassed with a prosthetic vascular graft $10 \mathrm{~mm}$ in diameter with rings (polytetrafluoroethylene; Gore-Tex, W. L. Gore \& Associates, Inc, Flagstaff, Ariz). The right BCV and the proximal portion of the SVC were ligated and cut, and extirpation of the tumor was completed without CPB. Operating time was 7 hours and blood loss was $1500 \mathrm{~g}$. The patient tolerated the procedures well and was monitored in the intensive care unit on the day of surgery. Her postoperative course was uneventful and she was discharged from the hospital on the 21 st postoperative day.

IMT was indicated by microscopic findings of the tumor, which showed a proliferation of spindle-shaped cells, mingled with chronic inflammatory infiltrate (Figure 2, A), pleomorphic tumor cells, which were scattered (Figure 2, $B$ ). Immunohistochemically, spindle-shaped cells were positive for alpha-smooth muscle actin (Figure 2, C), vimentin and HHF-35, but negative for anaplastic lymphoma kinase, desmin, S-100, CD 34, AE 1/AE 3, CAM 5.2, leukocyte common antigen (LCA), and CD 5, thus verifying the diagnosis of IMT.

\section{Discussion}

The name IMT evolved from the term "inflammatory psudotumor," which was initially reported in 1990 by Pettinato and colleagues. ${ }^{10}$ IMT includes a wide variety of histologic characteristics including benign to malignant.

The clinical behavior is generally considered to be indolent, and the symptoms depend on the location of the tumor that have been previously reported, such as soft tissues, respiratory tract, gastrointestinal tract, and so on. ${ }^{1}$ However, to the best of our knowledge, no IMT in the mediastinum eliciting the SVC syndrome has yet been reported. In the present case, the tumor aggressively surrounded mediastinal great vessels such as the SVC, right BCA, left CCA, and left SA and presented as SVC syndrome. At excision, the tumor could be dissected from the arteries; however, it was necessary to perform resection from the SVC to the right BCV due to severe adhesions.

Hematoxylin-eosin staining of the resected specimen showed the proliferation of spindle-shaped cells with inflammatory infiltrate and myxoid change. Immunohistochemical examination is 

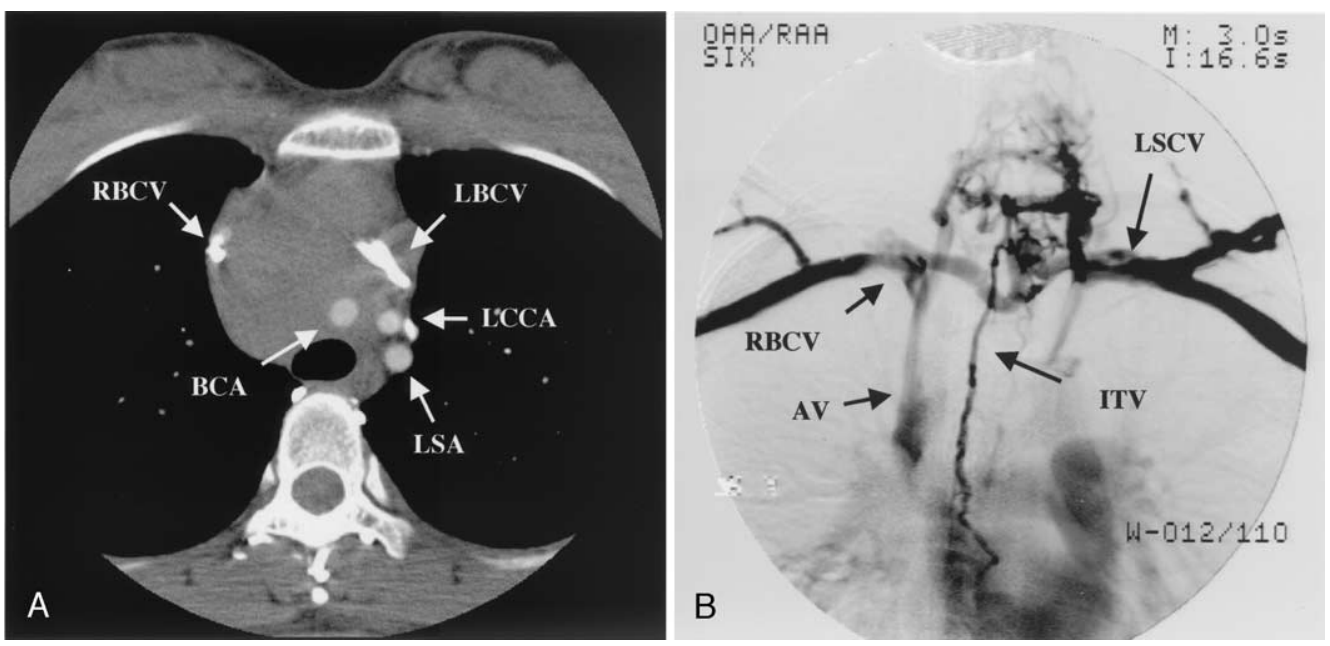

Figure 1. A, Chest CT performed preoperatively. The tumor involved the brachiocephalic vein (BCV), left brachiocephalic vein (LBCV), and right brachiocephalic artery (RBCA). The RBCV and LBCV were involved by the tumor and were nearly obstructed, but the lumen of the artery was well preserved. The tumor was closely adjacent to the left common carotid artery (LCCA) and the left subclavian artery (LSA). The trachea is also compressed by the tumor. B, Venography via the subcutaneous veins of the bilateral upper extremities. Note that the LBCV was not visualized in the early phase; note also the remarkable collateral drainage via the inferior thymic vein (ITV) and azygos vein (AV).
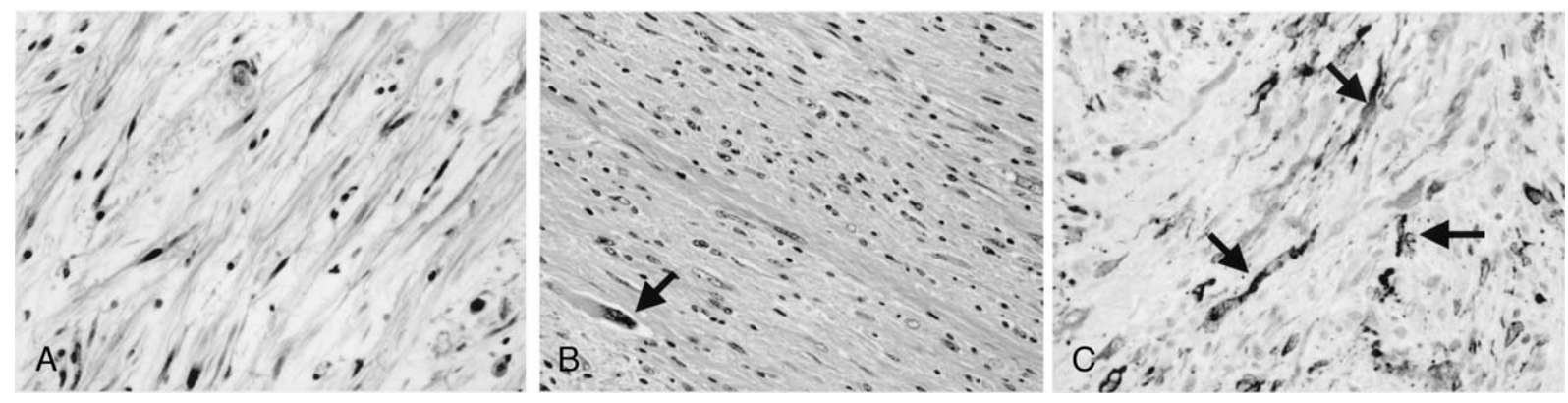

Figure 2. A, Hematoxylin-eosin staining at $\times \mathbf{2 0 0}$ magnitude. The tumor is made up of a proliferation of spindleshaped cells with inflammatory infiltrate and myxoid change. B, Hematoxylin-eosin staining at $\times 200$ magnitude. Spindle-shaped cells are arranged in fascicles, associated with collagenous stroma and chronic inflammatory cells. Tumor giant cells with pleomorphic nuclei are scattered (arrows). C, Alpha-smooth muscle actin was also positive on immunohistochemical staining in spindle-shaped cells (arrows).

helpful in diagnosing and differentiating between various types of sarcomas, and this case showed positive staining for alpha-smooth muscle actin and desmin, indicating a diagnosis of IMT. ${ }^{10}$ However, the presence of scattered tumor giant cells with pleomorphic nuclei in this case suggested low-grade malignancy.

The clinical presence of this case with SVC syndrome implicated the malignancy. Preoperative clinical diagnosis of this histologic type of tumor was impossible, and surgical excision seems to be essential for a good prognosis. Careful postoperative follow-up will be necessary for this case.

\section{References}

1. Coffin CM, Watterson J, Priest JR, et al. Extrapulmonary inflammatory myofibroblastic tumor (inflammatory pseudotumor). A clinicopatho- logic and immunohistochemical study of 84 cases. Am J Surg Pathol. 1995; 19:859-72.

2. Rubin BP. Recent progress in the classification of soft tissue tumors: role of genetics and clinical implications. Curr Opin Oncol. 2001;13: 256-60.

3. Yamamoto H, Watanabe K, Nagata M, et al. Inflammatory myofibroblastic tumor (IMT) of the pancreas. J Hepatobiliary Pancreat Surg. 2002;9:116-9.

4. Makhlouf HR, Sobin LH. Inflammatory myofibroblastic tumors (inflammatory pseudotumors) of the gastrointestinal tract: how closely are they related to inflammatory fibroid polyps? Hum Pathol. 2002; 33:307-15

5. Dennery MP, Rushton HG, Belman AB. Sonography for the detection and follow-up of primary nonsarcomatous bladder tumors in children. Urology. 2002;59:119-22.

6. Wenig BM, Devaney K, Bisceglia M. Inflammatory myofibroblastic tumor of the larynx. A clinicopathologic study of eight cases simulating a malignant spindle cell neoplasm. Cancer. 1995;76:2217-29. 
7. Tang TT, Segura AD, Oechler HW, et al. Inflammatory myofibrohistiocytic proliferation simulating sarcoma in children. Cancer. 1990; 65:1626-34

8. Amir R, Danahey D, Ferrer K, et al. Inflammatory myofibroblastic tumor presenting with tracheal obstruction in a pregnant woman. Am J Otolaryngol. 2002;23:362-7.
9. Li L, Cerilli LA, Wick MR. Inflammatory pseudotumor (myofibroblastic tumor) of the heart. Ann Diagn Pathol. 2002;6:116-21.

10. Pettinato G, Manivel JC, De Rosa N, Dehner LP. Inflammatory myofibroblastic tumor (plasma cell granuloma). Clinicopathologic study of 20 cases with immunohistochemical and ultrastructural observations. Am J Clin Pathol. 1990;94:538-46.

\title{
Intralobar sequestration: A rare cause of severe hemothorax
}

\author{
W. Wandschneider, MD, a and H. Illiasch, MD, ${ }^{b}$ Klagenfurt, Austria
}

A

22-year-old male patient was admitted to our emergency room with signs of severe hemorrhagic shock. Hemotocrit was $7.5 \%$ and red blood cell count was 2.7 million. After hemodynamic stabilization with plasma expanders and 3 units of packed red cells, the patient gave a history of sudden severe pain in the left hemothorax with dyspnea and growing dizziness. There was no trauma or signs of infection, no cough, and no hemoptysis. Emergency chest radiography showed an opaque left hemothorax with slight shifting of the mediastinum to the right.

Computed tomography (CT) scan showed pleural effusion in the left thoracic cavity and a mass in the median left lower lobe, which was suspicious of an intralobar sequestration. We therefore performed an angiography to assess the presence of this malformation and find the feeding vessel. The angiogram revealed a large vessel deriving from the abdominal aorta, perforating the left diaphragm, and supplying an intrapulmonary sequestration. A leak of contrast medium proved bleeding from this vessel into the pleural cavity (Figure 1). The vessel was immediately occluded with multiple coils. The control showed an occluded vessel with no contrast effusion (Figure 2).

After the occlusion the patient was taken to the operating room where a video-assisted thoracoscopy (VATS) was performed. Blood and thrombi were evacuated and the pleural cavity inspected. There was no more bleeding and the feeding artery could not be detected. A thoracoscopic resection of the median segment of the left lower lobe (segment 10) was performed.

Due to his blood loss, the patient postoperatively developed an acquired respiratory distress syndrome requiring 6 days of mechanical ventilation. The further course was uneventful; the patient

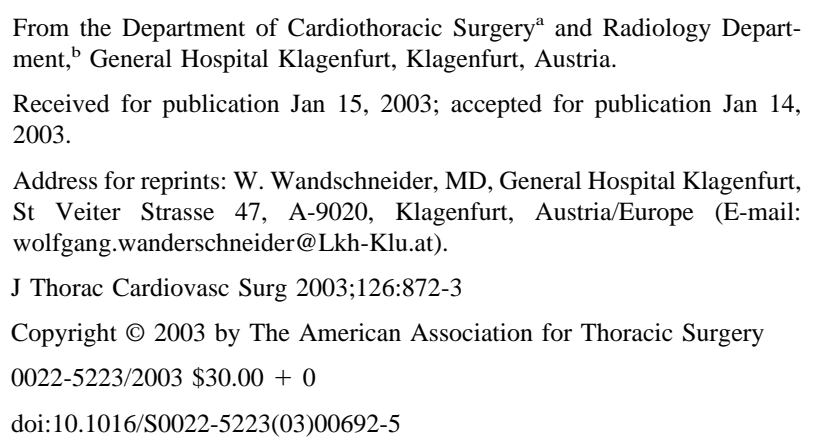

was transferred from the intensive care unit to the normal ward on day 9 and left the hospital on day 15. After 6 months his chest radiographs show adhesions in the left costophrenic sulcus with normal forced expiratory volume in 1 second, and the patient is currently without symptoms.

\section{Discussion}

A sequestration is a pulmonary malformation where pulmonary tissue is formed without a connection to the normal bronchial system, either within the lung (usually the lower lobe) or as a separate adjunct. Usually the sequestration has its own feeding artery deriving from the aorta. As sequestrations are most commonly located in the lower lobes, the artery frequently has its offspring from the abdominal aorta or splenic artery and perforates the diaphragm. As the systemic blood pressure in the sequestral artery is higher than the pulmonary artery pressure in the surrounding lung tissue, bleeding into the tissue is a well-known complication of this malformation. ${ }^{1,2}$ When the tissue communicates with a bronchus or bronchiolus, hemoptysis is possible. There are, however, only 2 reports in the recent literature ${ }^{1,3}$ of hemothorax as a consequence of bleeding into the pleural cavity.

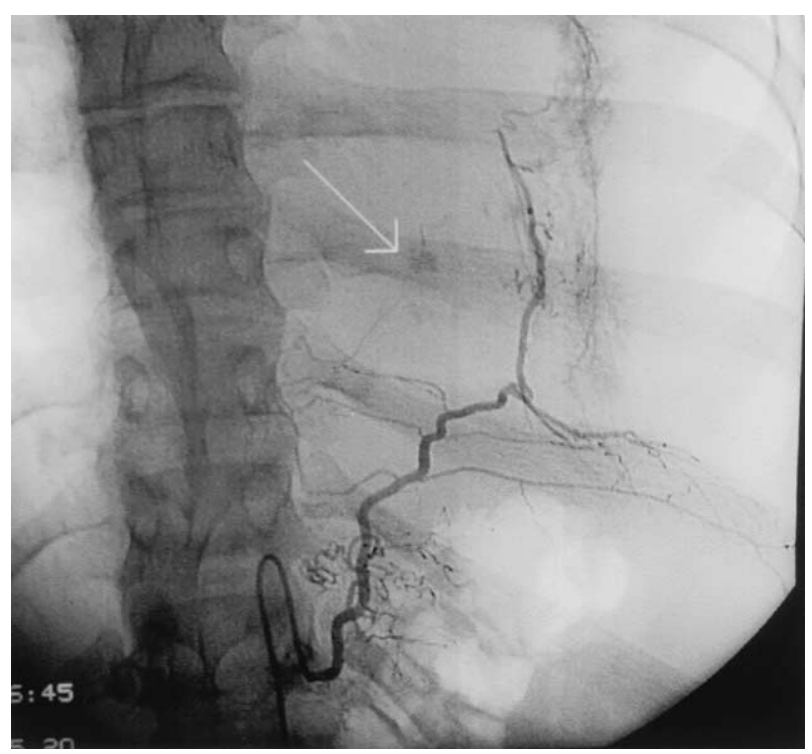

Figure 1. Angiography shows bleeding from the feeding vessel. 